\title{
Propuesta para implementar un sistema de gestión del conocimiento que apoye el diseño de un curso online
}

\author{
Proposal to implement a knowledge management system \\ to support the design of an online course \\ Cristian Sandoval Yáñez ${ }^{1}$ \\ Recibido 10 de enero de 2013, aceptado 26 de junio de 2013 \\ Received: January 10, 2013 Accepted: June 26, 2013
}

\begin{abstract}
RESUMEN
El objetivo de este artículo es presentar una propuesta de un sistema de gestión del conocimiento que entregue la orientación necesaria para la elaboración de un curso online. La propuesta está integrada por un modelo de capas que permite adquirir y almacenar el conocimiento y por un modelo informático que permite gestionar y transmitir el conocimiento al usuario.

Esta propuesta pretende fortalecer la creación de cursos online mejorando la calidad y permitiendo su reutilización en diferentes entornos virtuales de enseñanza-aprendizaje. Además responde a las tendencias actuales de la modalidad e-learning en las universidades de Chile, las que han incrementado considerablemente la creación de programas de estudio bajo esta modalidad de enseñanza.
\end{abstract}

Palabras clave: Educación a distancia, gestión del conocimiento, e-learning, cursos online, entorno virtual de enseñanza-aprendizaje.

\begin{abstract}
The aim of this paper is to present a proposal of a knowledge management system that delivers the necessary guidance for the development of an online course. The proposal consists of a layer model which allows for the acquisition and storage of knowledge and a computer model that allows the management and transferring of knowledge to the user.

This proposal aims to strengthen the creation of online courses to improve the quality and allowing their reuse in different virtual teaching and learning environments. It also responds to current trends of e-learning at universities in Chile, which have significantly increased the creation of curricula teaching this modality.

Keywords: Distance learning, knowledge management, e-learning, online courses, virtual teaching learning environment.
\end{abstract}

\section{INTRODUCCIÓN}

El proceso de aprendizaje es uno de los fenómenos más relevantes en la especie humana. Producto del aprendizaje las personas, en el transcurso de su existencia, cambian considerablemente sus conductas, actitudes, habilidades y capacidades producto de lo que aprenden en diferentes instancias de la vida. El aprendizaje formal está relacionado con los conocimientos que se adquieren mediante un proceso de aprendizaje dirigido por alguna institución de educación.

En la actualidad el aprendizaje formal ha evolucionado considerablemente por la intervención de diferentes actores complementarios que han provocado un

1 Escuela Universitaria de Educación Virtual. Universidad de Tarapacá. 18 de Septiembre 2222. Arica, Chile. E-mail: csandovy@uta.cl 
salto importante en relación con los paradigmas pedagógicos, la masificación de la educación y las nuevas formas de comunicarse.

La educación a distancia, las tecnologías de la información y comunicación (TIC), y la sociedad del conocimiento han provocado una evolución de la enseñanza que tiene como ejes relevantes, por un lado, el tiempo y espacio en el que se lleva a cabo el proceso de enseñanza-aprendizaje $y$, por otro lado, los nuevos roles que deben asumir tanto el profesor en su calidad de tutor y el estudiante como responsable de su aprendizaje.

La rápida expansión de internet ocurrida en todos los niveles de la sociedad también se ha reflejado en el ámbito educativo por medio de la educación a distancia, la que ha surgido al superar algunas de las limitantes que la educación tradicional no ha podido atender. Estas limitantes apuntan a: superar barreras sociales relacionadas al financiamiento del traslado y la permanencia por un largo tiempo del estudiante para poder responder al modelo de educación presencial, a permitir combinar el ámbito laboral con la educación, permitir el aprendizaje asíncrono sin la necesidad de coincidencia en el tiempo ni en el espacio con la acción docente y, finalmente, a mejorar la comunicación fuera del aula por medio del uso de las TIC. Debemos dejar en claro que estos avances no significan que la educación presencial dejará todo en manos de la modalidad a distancia, tampoco podemos pretender representar la educación a distancia como solo una herramienta más de aprendizaje que permite solucionar los problemas de la distancia, sino su vocación es constituir una metodología en sí misma, cuyos principios sean la creación de un método de aprendizaje flexible, en el cual el foco de atención recaiga sobre el estudiante.

El avance en las TIC ha provocado un cambio cultural en relación a la visión que se tiene de la modalidad a distancia, pero estas herramientas son solo el medio por el cual se desenvuelve esta modalidad. Hoy toma mayor relevancia la actualización de conocimientos del profesor y el aumento de autonomía que debe asumir el estudiante. El profesor no solo debe preocuparse de actualizar sus contenidos, sino esencialmente de motivar y lograr una comunicación fluida, clara, didáctica y entretenida, de manera de permitir al estudiante aprender, entendiendo que él pasa a ser el centro del modelo. Por lo tanto, la actualización docente es un desafío trascendental que ha surgido como respuesta a las nuevas demandas educacionales que se deben atender al momento de enfrentarse a un alumnado radicalmente opuesto al que se acostumbraba.

Por otro lado, la sociedad del conocimiento ha tomado una importancia relevante, ya que las organizaciones que incorporan y administran el conocimiento en su quehacer son capaces de ser competitivas y adaptarse a los cambios en el entorno. El objetivo principal del proceso de gestión del conocimiento consiste en mejorar la calidad de las decisiones que se toman en las organizaciones, esto se logra gracias a que se dispone de información fiable $\mathrm{y}$ válida que es entregada de manera oportuna y segura. Estos procesos permiten mejorar la eficiencia en las organizaciones debido a la masificación, almacenamiento y utilización del conocimiento en sus procesos productivos y administrativos, reduciendo la necesidad de redescubrirlo cada vez que se necesitan. Todos estos atributos pueden ser trasladados a instituciones de educación superior, responsables de generar y gestionar el conocimiento para la sociedad.

El presente trabajo tiene como objetivo primordial presentar una propuesta de sistema de gestión del conocimiento para apoyar al diseño de cursos online para la modalidad a distancia. Si bien es conocido que el diseño de un curso de estas características necesita del apoyo de un equipo multidisciplinario, la idea es presentar una propuesta que permita recopilar, almacenar y administrar el conocimiento explícito de cada disciplina con el propósito de apoyar a los tutores que pretendan migrar sus clases presenciales a un formato virtual.

\section{FORMULACIÓN DE LA PROPUESTA}

El desarrollo de las tecnologías de la información y comunicación parece entregarnos por fin las herramientas necesarias para realizar una efectiva enseñanza bajo la modalidad a distancia.

Farcas y Reininger [1] nos plantean que "La educación a distancia bajo la modalidad e-learning puede contribuir a la formación de los profesionales que el país precisa". Los autores demuestran, 
mediante estadísticas relacionadas con la elaboración de programas online, el notorio avance de la educación a distancia en los procesos de enseñanzaaprendizaje en nuestro país.

Una definición para la educación a distancia es la que plantea Moore citado por Eggers [2], quien la define como "una forma de aprendizaje planeada, que ocurre en un lugar diferente al de enseñanza y como resultado requiere técnicas especiales para diseñar cursos, técnicas de instrucción especializadas, métodos de comunicación electrónicos o por medio de otra tecnología, así como acuerdos administrativos y organizacionales especiales".

Es importante definir el concepto de e-learning y la relación que tiene con la educación a distancia, esto debido a que ambos conceptos se utilizan en diferentes apartados de este documento. Cabero [3] define modalidad e-learning como "una modalidad formativa a distancia que se apoya en la red, y que facilita la comunicación entre el profesor y los alumnos según determinadas herramientas sincrónicas y asincrónicas de la comunicación". Si bien las definiciones son semejantes, e-learning es básicamente la educación a distancia completamente virtualizada. Por lo tanto, podemos posesionar al e-learning como un caso particular de la educación a distancia.

Como complemento podemos decir que la educación a distancia se desarrolla en que una plataforma virtual, en la cual el estudiante se vincula a un curso online disponible en este medio. Estas plataformas, formalmente conocidas como entornos virtuales de enseñanza-aprendizaje, contienen un conjunto de herramientas tecnológicas que unidas a un modelo educativo permiten crear un ambiente que facilita el proceso de enseñanza-aprendizaje. El avance de estos entornos virtuales han disminuido considerablemente la distancia entre los actores principales (tutor y alumnos) involucrados en esta modalidad de enseñanza. Es importante tener en cuenta que las herramientas tecnológicas son solo un medio, pero por sí solas estas no podrán lograr un aprendizaje significativo en los alumnos si no se cuenta con un buen diseño del curso online, considerando que este curso es el nexo principal entre tutor y sus alumnos.

Diseñar un curso para ser enseñado en línea requiere una preparación especial y una consideración por el material. Además de los retos normales de definir el contenido y objetivos, un curso en línea requiere la utilización de un diseño instruccional eficiente y un diseño multimedial de calidad. Alanís [4] plantea que el diseño de un curso en línea requiere de un grupo de profesionales que apoye al instructor y lo ayude a generar un curso tecnológicamente viable, visualmente atractivo y pedagógicamente coherente.

Considerando lo planteado por el autor podemos definir que la creación de un curso online involucra un trabajo multidisciplinario, con distintas responsabilidades relacionadas con el diseño instruccional del curso, el diseño gráfico y multimedial, el modelo pedagógico que soporta el curso, el formato de estandarización $e$-learning para ser visualizado en diferentes entornos virtuales de enseñanza-aprendizaje y, finalmente, la evaluación del curso, para que pueda ser utilizado en el entorno virtual seleccionado. Todo este equipo debe estar al servicio del tutor experto, el que es responsable de recopilar toda la información y los contenidos del curso que se desea virtualizar.

Podemos asumir que los tutores expertos poseen distintos grados de conocimientos en tecnología de la información y distintos niveles de preparación para adaptar un curso presencial a un curso en modalidad a distancia, pero esta preparación no garantiza que el producto final sea portable y de calidad.

La calidad es un concepto complejo de definir y, dependiendo del contexto, subjetivo de evaluar. En el contexto de la modalidad a distancia, Tello [5] plantea que regularmente la evaluación de la calidad de los cursos virtuales se centra en el desarrollo y los resultados del curso, dejando de lado el diseño de este. El autor plantea la necesidad de "tener productos sobre los que tengamos certeza de su calidad antes de su difusión, es decir, productos que cumplan con determinados estándares de calidad, que evalúen su adecuada puesta en marcha".

Consideramos que la calidad de un curso online es un elemento fundamental en el éxito de la modalidad a distancia, tomaremos la métrica del diseño para asegurar que los cursos que se elaboren, utilizando el sistema de gestión del conocimiento propuesto, cumplan con los estándares de calidad necesarios.

La propuesta apunta a que cada vez que se desee crear un curso online no se comience desde cero 
o consultando de manera independiente a cada integrante del equipo multidisciplinario, sino que se comience del conocimiento generado por el sistema de gestión. Honeycutt [6] dice que la filosofía de la gestión del conocimiento es “entregar la información precisa para la persona apropiada en el instante oportuno, proporcionando herramientas para el análisis de esa información y la capacidad de responder a las ideas que se obtienen a partir de esa información".

Respondiendo a esta definición de gestión del conocimiento, el estudio presenta una propuesta concreta para la implementación de un sistema informático de gestión del conocimiento que sea el soporte necesario para crear, diseñar e implementar cursos online. Cabe destacar que su objetivo no es reemplazar el equipo multidisciplinario, sino pretende tomar la información de cada disciplina y proveer el conocimiento necesario para apoyar al tutor experto que desea crear un curso de estas características.

\section{CURSO ONLINE}

Es importante conocer la estructura de un curso online para poder definir la información que se requiere almacenar por la propuesta y así cumplir con la misión de generar el conocimiento necesario para apoyar la creación de este tipo de cursos. Fernández y Montes [7] han presentado un estudio en el que sintetizan los requerimientos necesarios para realizar el diseño y montaje de un curso online. Estos requerimientos apuntan a:

- Disponer de la tecnología requerida para la conexión a las intranet o internet.

- Formar un equipo de trabajo multidisciplinario que realice el diseño e implementación del curso.

- Establecer la organización del contenido del curso.

- Utilizar una adecuada presentación de las lecciones.

- Permitir el acceso a la bibliografía básica y complementaria sobre los temas de estudio.

- Disponer en la plataforma virtual de mecanismos de acceso sincrónico y asincrónico.

- Contar en la plataforma virtual con estadísticas de acceso y control.
De los requisitos presentados por los autores se rescatan los aspectos generales que debe tener un curso online elaborado a partir de la propuesta que se presenta en este documento. Por lo tanto se define un curso online como un paquete encapsulado que contiene cuatro elementos (Figura 1) que en su conjunto serán montados en algún entorno virtual de enseñanza-aprendizaje. Los elementos son: el programa del curso, las lecciones, las actividades de aprendizaje y el material bibliográfico y/o multimedial de apoyo al proceso de aprendizaje.

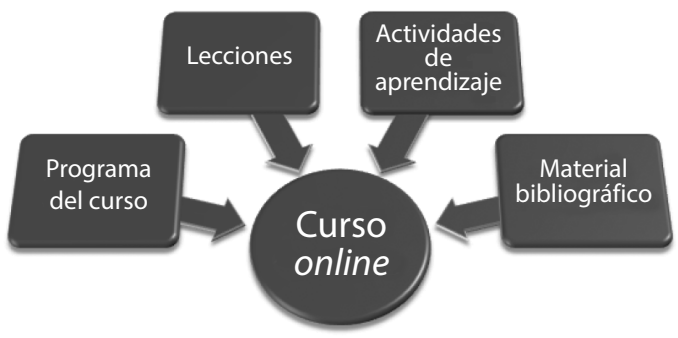

Figura 1. Esquema de elementos de un curso online para la propuesta. (Elaboración propia).

Del Carmen [8], realiza un estudio de los diferentes modelos de diseño instruccional que se deben considerar al momento de elaborar materiales para la modalidad online. La autora plantea que el papel del diseño instruccional en la modalidad online requiere de una buena planeación que prevea los objetivos a alcanzar y los medios necesarios para lograrlo. Textualmente plantea: "En la educación a distancia, los diferentes modelos de diseño instruccional pretenden clarificar, a quien recibe la instrucción, las formas de lograr el aprendizaje; y que no sea la distancia un impedimento para lograrlo". En otras palabras se busca claramente el objetivo de instrucción. Por estos motivos la propuesta pone énfasis en entregar el conocimiento necesario relacionado al diseño instruccional como base fundamental del diseño de un curso online.

\section{GESTIÓN DEL CONOCIMIENTO}

Antes de explicar la importancia de la gestión del conocimiento, vale la pena mencionar los conceptos que forman parte de esta frase. En primer lugar el concepto más sencillo de gestión es hacer que las cosas sucedan. Por otro lado el concepto de la palabra conocimiento se refiere a las creencias y 
compromisos, más allá de la mera información. $\mathrm{Si}$ consideramos que el conocimiento, en primera instancia, es creado en las mentes de las personas individualmente a partir de sus experiencias y competencias, y en una segunda instancia se comparte a partir del trabajo colaborativo entre las personas; la gestión será la encargada de identificar formas para fomentar y estimular el desarrollo de nuevos conocimientos, como también debe ser capaz de ofrecer formas efectivas de representar, organizar, reutilizar y renovar los conocimientos individuales y/o grupales.

Swan [9] nos dice que el reto de la gestión del conocimiento es transformar el conocimiento tácito en un conocimiento explícito. El mismo autor nos describe el conocimiento tácito como "un conocimiento que no puede articularse con facilidad y que, por lo tanto, solo existe en las manos y en las mentes de las personas, para manifestarse después mediante acciones", y el conocimiento explícito como "el conocimiento capturado y codificado en manuales, normas y procedimientos y resulta sencillo de transmitir". Por lo tanto podríamos afirmar que el conocimiento tácito es el inherente a las personas, producto de su experiencia, sabiduría y creatividad, en cambio el conocimiento explícito es el que se representa por un lenguaje formal y sistemático.

Drucker, citado por Concha [10], describe la importancia del conocimiento al catalogarlo como un valor agregado económico. El autor plantea que "nos estamos adentrando en la sociedad del conocimiento, donde el recurso económico clásico ya no es el capital, los recursos materiales, ni la mano de obra, sino que es y será el saber, donde los empleados de conocimiento desempeñarán un papel central".

Las palabras referenciadas en el párrafo anterior apuntan a la relevancia del conocimiento en el progreso de las organizaciones, las que han situado la gestión del conocimiento como eje central para convertir su capital humano en capital intelectual, lo que genera un valor agregado a la economía de la empresa. Pero hablar de gestión del conocimiento suele ser aún un tema confuso para los profesionales que trabajan en el área de educación en la modalidad e-learning. Para ello es necesario que las organizaciones educativas comprendan el valor que tiene la transferencia del conocimiento entre sus académicos, con lo que se logra optimizar recursos y mejorar la calidad de los procesos de enseñanza-aprendizaje.

En relación con vincular la gestión del conocimiento con la enseñanza superior, Schmelkes [11] realiza una reflexión sobre el traspaso de la gestión del conocimiento que se debería realizar desde las organizaciones productivas a las instituciones de educación superior; el autor explica que no es común encontrar instituciones de educación superior que se estén preocupando por el conocimiento que se puede o debe generar en ellas ni tampoco por su captura para la difusión.

Caicedo [12] escribe un artículo en el que explora el potencial de la gestión del conocimiento para su aplicación en la educación superior apoyada en las herramientas colaborativas. Respecto de lo antes expuesto, debemos hacer la siguiente consideración, una institución de educación superior constituye el paradigma de una organización especializada en la generación y manejo del conocimiento avanzado por sus grupos e institutos de investigación, pero ¿de qué sirve generar conocimiento si no se gestiona?

En el marco de la modalidad $e$-learning se destaca a Lozano [13], quien sostiene que "El buen e-learning, facilita y desarrolla la Gestión del Conocimiento en todas las organizaciones; si aprendemos a desarrollar el e-learning, encontraremos el mejor camino para hacer fluir el conocimiento en las organizaciones". Así, el aprendizaje debería ser constante y continuo, mediante cursos bien definidos y diseñados rápidamente para satisfacer una necesidad de formación específica, por lo tanto el desafío de la propuesta se centra en la calidad del diseño de los cursos como consecuencia de una buena gestión del conocimiento.

\section{MODELOS PARA LA GESTIÓN DEL CONOCIMIENTO}

El sistema de gestión del conocimiento que se propone en este estudio hace referencia a la teoría general de sistemas en lo que respecta a utilizar un modelo abstracto y medios tecnológicos para organizar, controlar y fomentar el conocimiento dentro de una organización. En la bibliografía consultada, sobre los modelos de gestión del conocimiento, se observan dos artículos presentados 
por Sánchez [14] y por Soto y Barrios [15] en los que se realizan estudios acabados de los modelos existentes.

Soto y Barrios realizan un análisis crítico del estado del arte de los modelos actuales. Los mismos autores citan a McElroy [16], quien describe dos generaciones (Figura 2) para la gestión del conocimiento, la primera se preocupa de los aspectos referentes a la distribución, diseminación y uso del conocimiento existente, mientras que la segunda se desarrolla sobre la base de la producción del conocimiento.

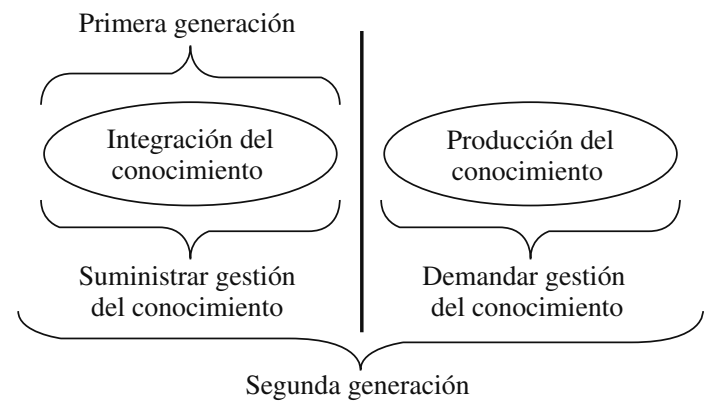

Figura 2. Primera y segunda gestión del conocimiento presentada por McElroy.

Puede afirmarse entonces que aunque exista una separación de las generaciones, la Figura 2 nos demuestra que se realizan acciones que pueden incluirse ambas generaciones, lo que significa que no es posible clasificar un modelo en alguna generación específica.

El modelo de gestión del conocimiento que se propone en este documento nace de un proceso híbrido que toma como referencia el modelo dinámico de rotación del conocimiento elaborado por Goñi [17], y el modelo de integración tecnológica planteado por Kerschberg [18]. Cabe destacar que ambos modelos de gestión están centrados en organizaciones empresariales y no educativas, motivo por el cual no se consideran en forma independiente como opción de solución para la propuesta planteada en este estudio.

El modelo dinámico de rotación del conocimiento (Figura 3) se centra en un conjunto de acciones $\mathrm{u}$ operaciones que se deben realizar de manera recursiva para lograr la rotación del conocimiento en una organización.

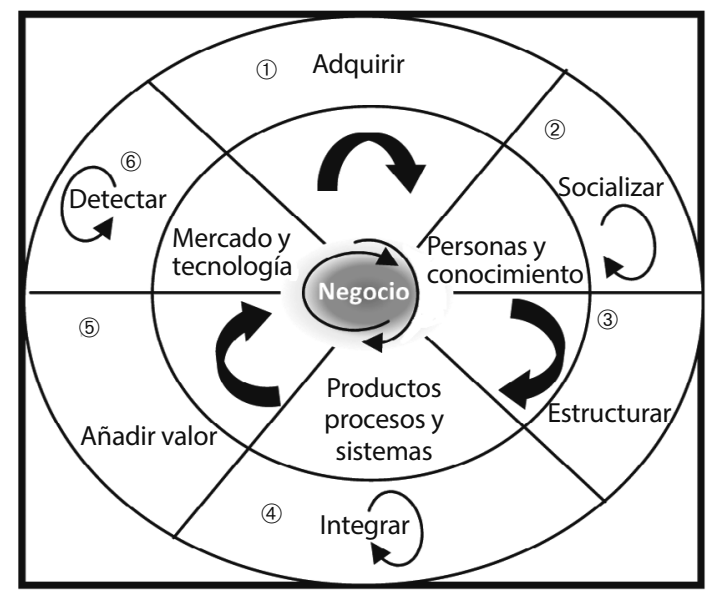

Figura 3. Modelo dinámico de rotación del conocimiento presentado por Goñi.

Las acciones del modelo son: adquirir conocimiento del entorno por medio de desarrollar labores activas, socializar el conocimiento por medio de la transformación del conocimiento tácito en un conocimiento explícito, estructurar el conocimiento utilizando tecnología, integrar el conocimiento por medio de la combinación de sistemas para transferir el conocimiento, añadir valor al conocimiento cuando se logran los objetivos planteados, lo que permite obtener un valor superior al costo de adquisición y transformación del conocimiento y, finalmente, detectar las oportunidades que ofrezca el conocimiento.

Si bien el modelo planteado por Goñi [17] presenta un conjunto de acciones que permiten la generación y almacenamiento del conocimiento, no posee una integración tecnológica que permita la automatización de todas las acciones y la conexión remota de los tutores que serán los usuarios del sistema. El modelo plantea un entorno cerrado en una organización y solo gestiona el conocimiento en personas sin incluir otros tipos de fuentes generadoras de conocimiento.

El modelo de integración tecnológica presentado por Kerschberg es un modelo con un marcado enfoque tecnológico que reconoce la heterogeneidad de las fuentes de información que alimentan el conocimiento. El autor establece una división de capas con gran integración, lo que permite una buena comunicación y un gran dinamismo entre los componentes al momento de gestionar el 
conocimiento. Las capas que identifica el autor son: presentación, gestión del conocimiento y fuentes de información (Figura 4).

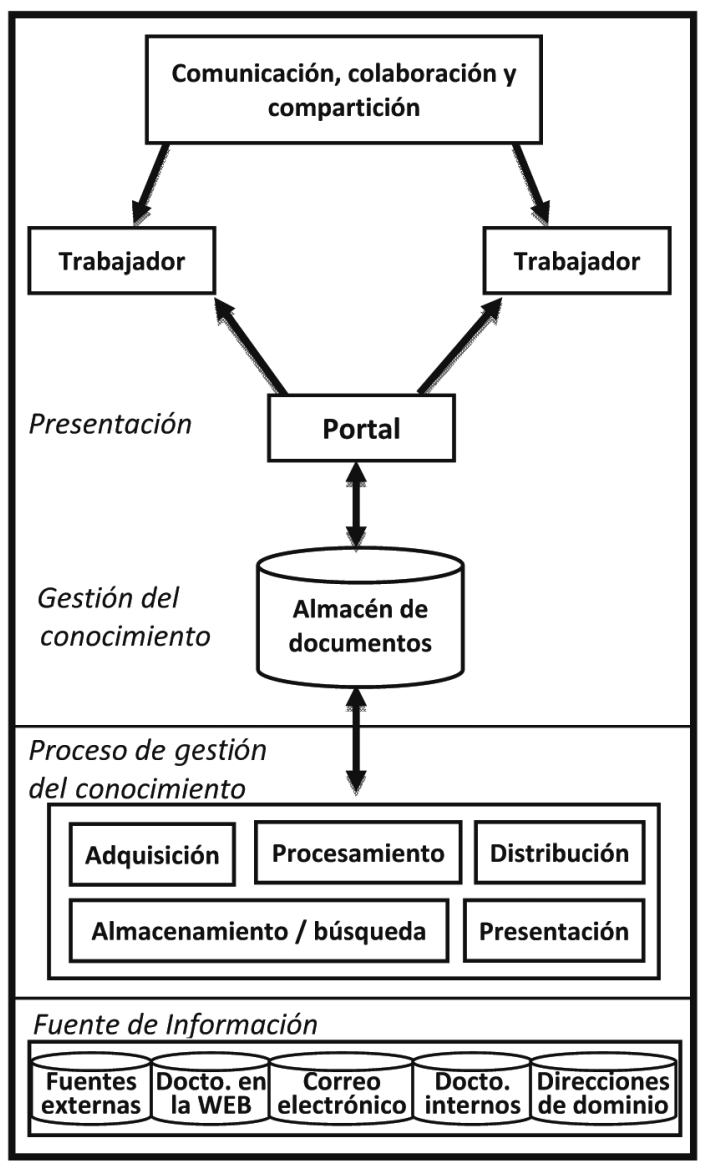

Figura 4. Modelo de integración tecnológica presentado por Kerschberg.

El modelo presentado por Kerschberg, si bien reconoce otras fuentes de generación del conocimiento e integra tecnología, no presenta acciones fundamentales para asegurar la calidad del curso virtual. Estas acciones tienen relación con la retroalimentación que debe recibir el sistema de parte de los usuarios y la sociabilización del conocimiento antes de ser presentado a los usuarios. $\mathrm{Si}$ bien ambos modelos estudiados tienen una orientación empresarial, sus aportes se utilizarán como base de la propuesta, a la que se le agregará el enfoque pedagógico necesario para asegurar la calidad del diseño del curso.

Eggers [2], Tello [5] y Llarena [19] realizan estudios en los que definen métricas de aseguramiento de la calidad para cursos online. Los autores elaboran mediciones para el proceso completo de este tipo de cursos, es decir, consideran la creación, el desarrollo y la evaluación del curso. Para nuestra propuesta solo nos centraremos en las métricas relacionadas a la creación del curso. Los autores apuntan al diseño instruccional como factor crítico para evaluar la calidad del diseño de un curso de estas características. Las métricas asociadas a este factor clave las dividen en: la estructura, el diseño, los contenidos, y la metodología utilizada para el desarrollo del curso.

A continuación se presenta la propuesta de sistema de gestión del conocimiento que toma las acciones del modelo de Goñi, la integración tecnológica de Kerschberg, las métricas de aseguramiento de la calidad y una innovación que soporte el factor pedagógico para el diseño de un curso online.

\section{PROPUESTA DE SISTEMA DE GESTIÓN DEL CONOCIMIENTO}

La propuesta del sistema de gestión del conocimiento se divide en dos modelados (Figura 5): el primero, llamado modelo de capas que está orientado a dividir en capas los componentes y describir sus funcionalidades y, el segundo, un modelo informático orientado a realizar el análisis informático para la automatización del sistema propuesto.

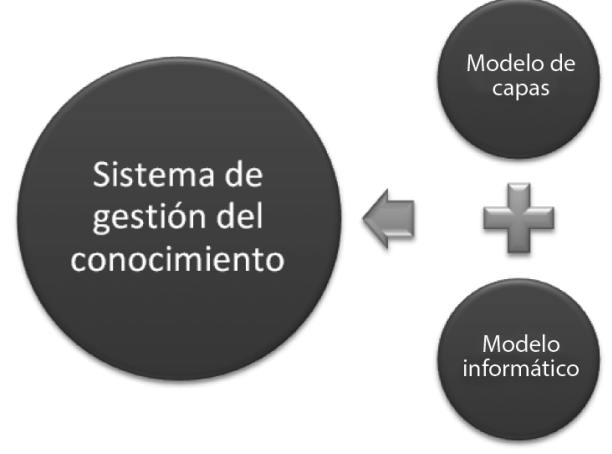

Figura 5. Modelos que soportan la propuesta del Sistema de Gestión del Conocimiento. (Elaboración propia).

El concepto de capas es rescatado del Modelo de Referencia de Integración de Pressman [20], en el que definen las capas como una forma lógica de ordenar y agrupar componentes que tiene relación entre sí. El autor modela un software a partir de 
una división de capas para facilitar la integridad y el mantenimiento de los componentes.

\section{Modelo de capas}

La primera fase está orientada a elaborar un modelo para la gestión del conocimiento que sea capaz de adquirir el conocimiento tácito y transformarlo en un conocimiento explícito. Una vez transformado el conocimiento se almacenará para quedar disponible, mediante un sistema informático, para ser utilizado por aquellos tutores que necesiten apoyo en el diseño de un curso online.

El modelo que se elaboró da respuesta a los siguientes pilares fundamentales del estudio:

- La gestión eficaz del conocimiento requiere una sinergia entre los profesionales encargados de generar la información.

- La gestión del conocimiento requiere de un soporte tecnológico que permita a los profesionales almacenar y administrar el conocimiento.
- La gestión del conocimiento es un proceso de mejora continua, por lo que se requiere compartir, mantener, reutilizar y actualizar el conocimiento.

Estos pilares se sustentan en una arquitectura de capas tomada del modelo Integración tecnológica, que divide las fuentes de información del proceso de gestión del conocimiento y de la presentación del conocimiento generado.

La separación de capas es solo una metáfora, ya que el modelo se sustenta en la comunicación entre las capas como base fundamental que permite la generación, utilización y actualización del conocimiento. Otros aspectos tomados del modelo de integración son la diversidad de las fuentes de información y el uso de las TIC.

El modelo elaborado (Figura 6) utiliza como eje central un proceso de gestión del conocimiento que nace de algunos procesos tomados del modelo Rotación del conocimiento, estos procesos permiten

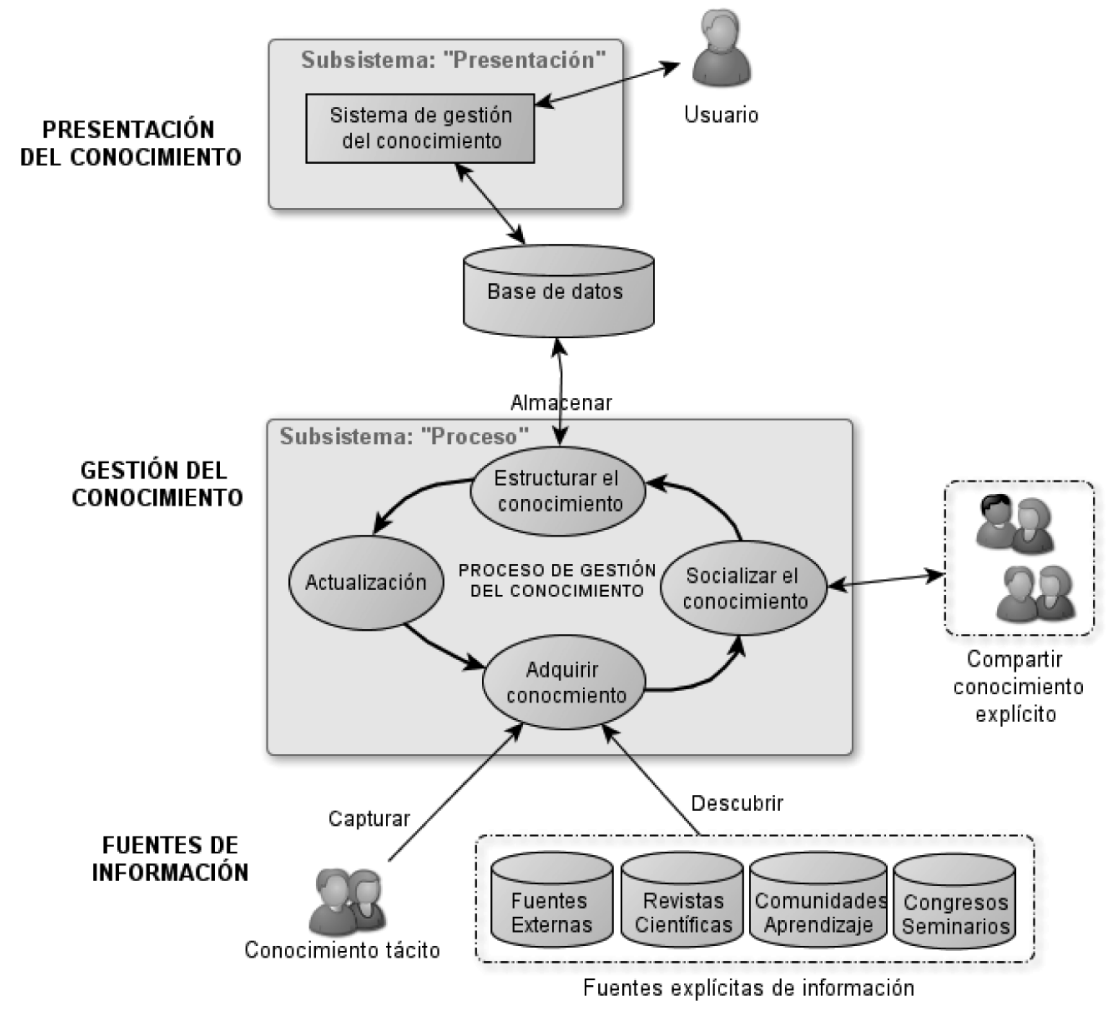

Figura 6. Modelo de capas para el Sistema de Gestión del Conocimiento que apoya el diseño de cursos online. (Elaboración propia). 
generar el conocimiento desde las distintas disciplinas involucradas en el diseño de un curso online.

\section{-Capa "Fuentes de Información"}

El proceso que inicia al sistema está relacionado con la captura y el descubrimiento de la información relacionada con las distintas disciplinas necesarias para crear un curso online.

\section{Capas y componentes}

El modelo propone tres capas que representan el ciclo evolutivo de la generación del conocimiento desde la captura del conocimiento tácito que posee cada uno de los profesionales expertos en el diseño de cursos online, pasando por la transformación a un conocimiento explícito, para que finalmente se genere el conocimiento necesario para que el usuario lo utilice cuando lo necesite.

En esta capa resulta imprescindible la formación de un equipo multidisciplinario integrado por especialistas en el diseño de un curso online que aporten las diferentes temáticas necesarias para cumplir con el objetivo de ingresar el conocimiento tácito. Entre los diversos roles que se pueden presentar en un equipo de educación a distancia, podemos mencionar aquellos que tiene directa relación con el diseño de un curso online y que son necesarios para el modelo del sistema de gestión propuesto, por este motivo no se incluye en este equipo al tutor experto en contenido, ya que es quien utilizará el sistema propuesto. Los roles de la propuesta son:

- Coordinador general: Profesional organizador del equipo, los procesos y las tareas que se deben cumplir para llevar a delante el proceso de diseño. Define la planificación estableciendo un cronograma de actividades, además de proponer y controlar las tareas a realizar por cada uno de los integrantes. Responsable del normal funcionamiento del diseño del curso online.

- Asesor de diseño instruccional: Profesional especialista en el diseño didáctico del curso online encargado de asesorar al profesor en el diseño de las actividades de enseñanza aprendizaje y en el diseño de las estrategias didácticas que se deben aplicar en el curso.

- Soporte tecnológico: Apoya al equipo docente seleccionando herramientas tecnológicas adecuadas para el logro, por parte del alumno, de los objetivos de aprendizaje propuestos. Se preocupa de definir los estándares e-learning y de la instalación del curso en la plataforma virtual para que los alumnos puedan acceder a él.

- Diseñador multimedia: Elabora productos audiovisuales que interpretan los requerimientos del tutor del curso con el fin de enriquecer y complementar la enseñanza de los alumnos a distancia. Es el encargado de mediatizar los contenidos. Además selecciona los recursos gráficos y multimediales adecuados para los cursos online.

- Experto en teorías del aprendizaje: Profesional encargado de entregar conocimientos sobre las tendencias o teorías del aprendizaje relacionadas a la educación a distancia o su adaptación a esta modalidad.

- Evaluador del curso: Tiene a su cargo la evaluación del curso online antes de ser levantado a una plataforma virtual, también propone medidas correctivas para solucionar inconvenientes que se hayan producido durante la creación del curso.

La efectividad de la enseñanza a distancia está directamente relacionada a la calidad del diseño de un curso online y a la mediatización efectiva que se produce durante el desarrollo del curso. Tanto los diseñadores del curso como el tutor deben tener la capacidad de utilizar en forma estratégica y creativa múltiples productos multimedia que permitan canalizar la comunicación sincrónica o asincrónica con el objetivo de lograr un aprendizaje significativo en los alumnos. Por otra parte es necesario que los diseñadores de los cursos online analicen las características cognitivas del alumno, sus conocimientos previos, su motivación, su realidad tecnológica y el contexto social en el que se encuentran.

Una vez definido el equipo multidisciplinario, cada uno de sus actores tendrá la responsabilidad de entregar información actualizada de las tendencias para la modalidad e-learning en cada una de sus disciplinas. Este conocimiento tácito será complementado a la información que se pueda 
descubrir de las fuentes explícitas de información, como comunidades de aprendizaje, base de datos bibliográficas, fuentes externas, revistas científicas, seminarios y congresos. Los aportes del equipo multidisciplinario y la información de las fuentes explícitas serán entregadas a la capa encargada de procesar el conocimiento en el modelo propuesto.

\section{-Capa "Gestión del conocimiento"}

La capa se centraliza en el proceso de gestión del conocimiento, que se encarga de transformar la información tácita por medio de la interacción con el equipo multidisciplinario, en conocimiento explícito para luego almacenarlo en una base de datos, con el fin de proporcionar el conocimiento a la capa de presentación del conocimiento. Este proceso de gestión se divide en cuatro actividades:

- Adquirir conocimiento: Actividad en la que se captura la información tácita elaborada por el equipo multidisciplinario y se incluye información descubierta desde las fuentes explícitas de información. Esta actividad puede ser automatizado y finaliza una vez que la información pasa a ser explícita para el proceso. El responsable de esta actividad es el coordinador general o en su defecto cualquier integrante del equipo multidisciplinario.

- Socializar el conocimiento: Esta actividad tiene como objetivo socializar la información explícita, con el fin de compartirla con los integrantes para que de acuerdo con un trabajo colaborativo se dialogue y debatir y producir los nexos entre las diferentes disciplinas, lo que permite a partir de la información compartida generar el conocimiento.

- Estructurar el conocimiento: Actividad automatizada que permite almacenar, en un gestor de base de datos, el conocimiento explicito elaborado en la actividad anterior.

- Actualización: Esta actividad tiene como propósito evaluar los resultados de la implementación de los cursos online con el fin de retroalimentar al proceso, y así generar un nuevo ciclo de las fases de gestión cuando corresponda. En esta actividad se deben elaborar encuestas que permitan rescatar indicadores de gestión para realizar un seguimiento, evaluación y análisis del proceso.

\section{-Capa "Presentación del conocimiento"}

En esta capa está disponible el sistema informático que transforma el conocimiento implícito en conocimiento explicito para que el usuario (tutor) tenga el apoyo necesario al momento de diseñar un curso online. Esta plataforma tecnológica pretende entregar, por medio del conocimiento almacenado, la capacidad y experiencia del equipo multidisciplinario que está almacenada en la base de datos, para permitir generar, acceder, almacenar y transferir el conocimiento en la institución de educación superior.

La idea central es que el conocimiento no se utilice y no se pierda tiempo en generar conocimiento que ya existe. El uso de este conocimiento permitirá que los cursos online se elaboren con un nivel de calidad que permita un mejor entendimiento de los alumnos, y como consecuencia la disminución de la deserción en este tipo de modalidad. Como valor agregado podemos mencionar que un curso online elaborado a base de esta propuesta puede romper los prejuicios culturales que desacreditan esta modalidad de enseñanza en nuestro país.

\section{Modelo informático}

La segunda fase está orientada al diseño informático del sistema, que toma como referencia el modelo de capas y así descubrir las necesidades y servicios que el sistema debe proveer a sus usuarios. Esta segunda fase finaliza con la elaboración del diagrama de casos de uso, basado en el lenguaje UML [21], de los requerimientos que se definieron para el sistema.

\section{A. Requerimientos de sistema}

Los requerimientos son una descripción de las necesidades o servicios que el sistema debe proveer para sus futuros usuarios. El objetivo principal de esta etapa es identificar y documentar los requerimientos con un lenguaje natural que fácilmente puedan ser comprendidos por los clientes que necesitan el sistema, usuarios que utilizarán el sistema y el equipo informático que en un futuro debe implementar el sistema en cuestión. Tomando como referencia el modelo elaborado en este estudio, y en particular los subsistemas que se deben automatizar, a continuación se definen los requerimientos necesarios para cumplir con las necesidades del sistema. 
- El sistema debe permitir la creación de diferentes tipos de usuarios, como el tutor, los integrantes del equipo multidisciplinario y un administrador del sistema.

- El sistema debe permitir autentificar el ingreso de los usuarios con sus respectivos perfiles.

- El sistema debe permitir mantener la solicitud de información para las distintas disciplinas que registrarán información en el sistema.

- El sistema debe permitir el ingreso y administración de la información que captura de las diferentes disciplinas necesarias para el diseño de un curso online.

- El sistema debe permitir el trabajo colaborativo de los integrantes del equipo multidisciplinario para permitir socializar el conocimiento.

- El sistema deberá permitir elaborar y realizar encuestas, además de generar indicadores para evaluar el diseño de los cursos online y la aceptación del sistema por parte de los tutores.
- El sistema debe entregar el conocimiento explícito a los tutores que deseen diseñar un curso online.

- El sistema debe proveer un ambiente en el que el tutor pueda diseñar y visualizar el curso online en el que está trabajando.

\section{B. Análisis del sistema}

Esta etapa está dedicada a elaborar un diagrama de casos de uso (Figura 7), el cual se elabora a partir de los requerimientos funcionales definidos para el sistema.

Cabe destacar que el diagrama de casos de uso no es un diagrama que demuestre secuencialidad entre sus casos de uso, sino que modela los actores que participan en el sistema y los servicios a los que tiene acceso cada uno de estos actores. Además se modela la relación que existe entre los servicios, entre los actores, como también entre actores y servicios que presta el sistema.

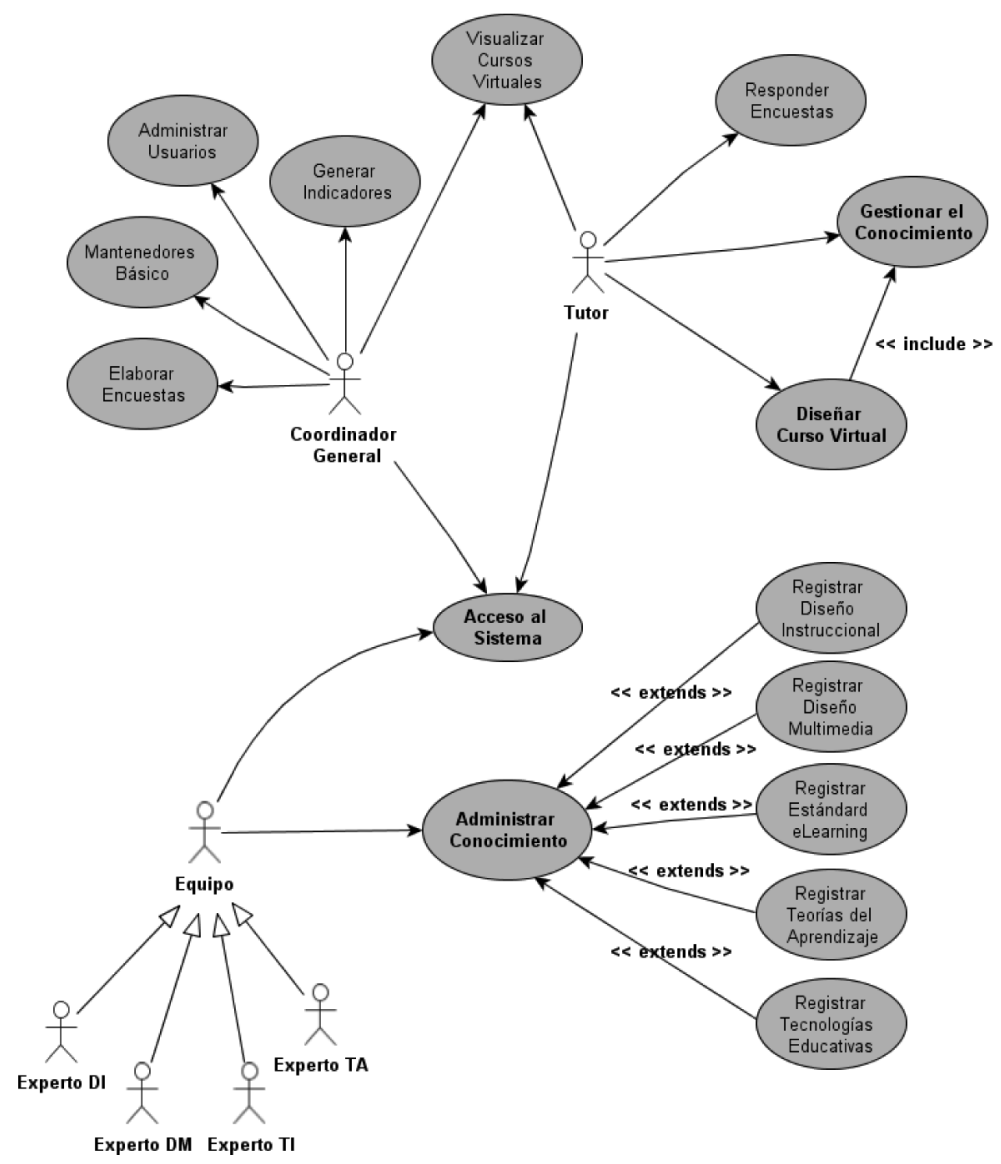

Figura 7. Diagrama de casos de uso para la propuesta del sistema. (Elaboración propia). 


\section{Definición de actores}

Cada actor tiene definido un conjunto de actividades que puede realizar en el sistema, estas son:

\section{-Actor coordinador general}

- Responsable de crear los usuarios del sistema.

- Responsable de utilizar los mantenedores de información básica.

- Encargado de registrar las encuestas en el sistema.

- Encargado de supervisar los cursos que fueron diseñados en el sistema.

- Elabora indicadores que permiten realizar una retroalimentación a cada usuario del sistema.

\section{-Actor tutor}

- Acceso a una aplicación donde realiza consultas relativas a la elaboración de un curso online.

- Elabora un curso online con apoyo del conocimiento almacenado en el sistema.

- Visualiza el curso elaborado.

- Responde encuestas relativas al sistema.

\section{-Actor experto DI}

Registra información relacionada con el diseño instruccional necesario para la gestión del conocimiento.

\section{-Actor experto DM}

Registra información relacionada con el diseño multimedial necesaria para la gestión del conocimiento.

\section{-Actor experto TI}

Registra información relacionada con las tecnologías de la información, tecnología educativa instruccional y plataformas e-learning necesarias para la gestión del conocimiento.

\section{-Actor experto TA}

Registra información sobre las teorías del aprendizaje y sus nuevas tendencias relacionadas con la educación a distancia, necesarias para la gestión del conocimiento.

\section{-Actor equipo}

Rol genérico que permite asociar a los actores que pertenecen al equipo multidisciplinario encargado de entregar la información, según su experticia necesaria para alimentar al sistema y producir el conocimiento.

\section{Definición de casos de uso}

Cada caso de uso tiene definido un conjunto de actividades que puede realizar, representados como casos de uso, estos son:

\section{-Administrar usuarios}

Servicio que permite crear un usuario para que pueda utilizar el sistema.

\section{-Acceso al sistema}

Servicio que permite autentificar la persona que está ingresando al sistema.

\section{-Mantenedores básicos}

Un mantenedor es un proceso que permite ingresar, modificar y eliminar información relacionada con alguna temática del sistema. Un ejemplo se produce cuando es necesario crear un nuevo perfil de usuario que no existe.

\section{-Elaborar encuestas}

Servicio que permite crear encuestas relacionadas al aporte del sistema al diseño de un curso online.

\section{-Generar indicadores}

Servicio que a base de las encuestas efectuadas realiza un análisis de diferentes variables relacionadas a la satisfacción y calidad en la elaboración de cursos online.

\section{-Administrar conocimiento}

Módulo que permite ingresar la información tácita, para almacenarla y transformala en conocimiento explícito. Este módulo posee diferentes interfaces, las que permiten registrar el diseño instruccional, el diseño multimedia, los estándares e-learning y las teorías del aprendizaje.

\section{-Registrar diseño instruccional}

Servicio que permite ingresar información tradicional y las nuevas temáticas relacionadas al diseño instruccional que debería tener un curso online. Esta información es base para generar conocimiento.

\section{-Registrar diseño multimedia}

Servicio que permite ingresar información tradicional y las nuevas temáticas relacionadas al diseño multimedia para elaborar productos que apoyen el aprendizaje útil para un curso online. Esta información es base para generar conocimiento. 


\section{-Registrar estándar e-learning}

Servicio que permite ingresar información tradicional y las nuevas temáticas relacionadas a los estándares e-learning con el se puede empaquetar un curso online. Esta información es base para generar conocimiento.

\section{-Registrar teorías del aprendizaje}

Servicio que permite ingresar información tradicional y las nuevas temáticas relacionadas las teorías del aprendizaje relacionadas al modelo de educación a distancia. Esta información es base para la generar conocimiento.

\section{-Diseñar curso online}

Aplicación que permite ingresar y validar, según el conocimiento explícito almacenado en el sistema, las estructura del curso online que consta de las siguientes partes: aspectos generales, contenidos, evaluaciones y bibliografía.

\section{-Gestionar el conocimiento}

Aplicación que entrega el conocimiento al usuario encargado de diseñar un curso online. Por medio de ejemplos y respuesta a preguntas frecuentes el sistema guiará y responderá consultas que permiten llevar a cabo el apoyo al diseño.

\section{-Responder encuestas}

Servicio que permite ejecutar encuestas relacionadas al sistema de gestión del conocimiento propuesto.

\section{-Visualizar curso online}

Aplicación que permite visualizar los cursos online que se han diseñado, tal como se verían en una plataforma e-learning.

\section{CONCLUSIONES}

Para asegurar el éxito de un curso online es necesario que los alumnos capten las ventajas pedagógicas y didácticas ofrecidas en este tipo de enseñanza. Pero estas ventajas no pueden cimentarse solamente en la facilidad de trabajar en espacios diferentes y tiempos asíncronos. Tópicos como las estrategias de comunicación e interacción tutor-estudiante, el desarrollo multimedial basado en animaciones, video, audio, simulaciones y objetos de aprendizaje, la teoría de aprendizaje sobre la que se guía el diseño del curso y el diseño instruccional relacionado con la evaluación de los aprendizajes; en su conjunto toman un valor agregado muy importante, el que es necesario evaluar antes de iniciar el diseño de un curso online para alcanzar óptimos niveles de calidad.

No todos los tutores poseen habilidades para diseñar cursos o enseñar en modalidad e-learning, tampoco puede esperarse que sepan intuitivamente cómo diseñar y dirigir con éxito un curso online; es muy importante proporcionarles el entrenamiento y apoyo necesario. La estrategia de asociar la gestión del conocimiento mediante un sistema informático es una manera innovadora y efectiva de apoyar a los tutores para que se desenvuelvan de buena manera en este desafío.

Pensar que un curso online se diseña colocando literalmente en la plataforma $e$-learning el programa y los contenidos de una clase tradicional resulta un grave error. El diseño se trata de una tarea más compleja, que exige a los tutores aprender nuevas habilidades tecnológicas, otras formas de organizar contenidos, nuevas formas de evaluar, manejo de herramientas de autor, manejo de plataformas e-learning y un nuevo estilo de enseñanza basado en la teoría constructivista del aprendizaje.

El sistema propuesto si bien no asegura completamente la calidad del diseño de un curso en esta modalidad, sí entrega buenas prácticas y estándares necesarios que permiten el diseño de calidad.

Es importante destacar que por las encuestas, proporcionadas por el sistema propuesto, se puede conocer la percepción de los alumnos y docentes, lo que permite mejorar el diseño de nuevos cursos, potenciando los aspectos señalados como positivos y mejorando los que así lo requieran.

Hasta hace muy poco tiempo la mayoría de los programas y productos informáticos de carácter educativo eran desarrollados por programadores y diseñadores profesionales; los docentes desempeñaban un discreto rol como expertos en contenidos. $\mathrm{La}$ propuesta de este estudio busca suministrar un sistema informático donde los tutores tengan el apoyo necesario al momento de crear los programas, contenidos, material de apoyo y evaluaciones. Todos estos factores pueden influir significativamente en la calidad e impacto de la enseñanza. 
Finalmente, la incorporación de la gestión del conocimiento al proceso de diseño de un curso online facilita las consultas periódicas sobre la información almacenada que ha sido generada por un equipo multidisciplinario, de esta forma se puede utilizar ese conocimiento en función de solucionar los problemas que se presenten y favorecer el perfeccionamiento de los métodos de trabajo para mejorar los tiempos de elaboración y los resultados esperados al momento de diseñar un curso para la modalidad online.

\section{REFERENCIAS}

[1] D. Farcas and M. Reininger. "Distance Education in the Form of E-Learning in Chile: Training Human Capital for the 21st Century". International Journal of Advanced Corporate Learning. Vol. 3, Issue 3, pp. 14-18. August, 2010. ISSN: 18675565. DOI: 10.3991/ijac. v3i3.1372.

[2] K. Eggers. "Criterios para evaluar la calidad de la dimensión pedagógica del sistema de Educación a distancia de la UDLA". Congreso LatinEduca 2005. La Habana, Cuba. Noviembre 2005.

[3] J. Cabero. "Bases pedagógicas del e-learning". Revista de Universidad y Sociedad del Conocimiento (RUSC). Vol. $3 \mathrm{~N}^{\mathrm{o}}$ 1, p. 2. Abril 2006. ISSN: 1698-580X. Fecha de Consulta: 12 de junio 2012. URL: http:// www.uoc.edu/rusc/3/1/dt/esp/cabero.pdf

[4] M. Alanís. "Preparando cursos en línea para ser impartidos por internet”. Primer Congreso Virtual Latinoamericano de Educación a Distancia. Marzo-Abril 2004.

[5] I. Tello. "Modelo de evaluación de la calidad de cursos formativos impartidos a través de internet". AIESAD RIED. Vol. $13 \mathrm{~N}^{\circ} 1$, pp. 209-240. 2010.

[6] J. Honeycutt. "Así es la Gestión del Conocimiento". Mcgraw-Hill/Interamericana de España, S.A. 1ra Edición. España. Vol. 1, p. 16. 2001. ISBN: 9788448129118.

[7] C. Fernández y M. Montes. "Aspectos a garantizar en la confección de cursos virtuales". Congreso LatinEduca 2004. La Habana, Cuba. Marzo 2003.

[8] A. Del Carmen. "Investigación documental: El Diseño Instruccional en la Educación a Distancia Un Acercamiento a los Modelos".
Revista de Innovación Educativa. Vol. 1 $\mathrm{N}^{\circ}$ 10, pp. 104-119. Abril 2009. ISSN: 16656180.

[9] J. Swan, M. Robertson y S. Newell. "Gestión del conocimiento: el Factor Humano". Editorial Thomson. Madrid, España. pp. 215233. 2002.

[10] M. Concha. "E-LEARNING: El aprendizaje a distancia es la herramienta por excelencia para la empresa del siglo XXI", pp. 1-4. Fecha de Consulta: 15 Agosto 2012. URL: http://www.petroleoyv.com/website/uploads/ CONCHA_3.pdf

[11] C. Schmelkes. "Reflexiones sobre la gestión del conocimiento en las Instituciones de Educación Superior". Administración y Organizaciones. Vol. 13 No 26, pp. 81-91. Enero 2011. ISSN: 1665014X.

[12] J. Caicedo. "La Gestión del conocimiento y las herramientas colaborativas: una alternativa de aplicación en Instituciones de educación superior". Revista de Investigación. Vol. 1 No 71, pp. 11-31. 2010. ISSN: 10102914.

[13] J. Lozano, G. Spitz y N. Van Dam. "Implementar e-Learning o Gestión del Conocimiento". Fecha de Consulta: 17 Agosto 2012. URL: http://www.learningreview.com/articulosy-entrevistas-gestion/219-implementar- $e$ learning-o-gestiel-conocimiento

[14] M. Sánchez. "Breve inventario de los modelos para la gestión del conocimiento en las organizaciones". Acimed. Vol. 13 No6, pp. 1-18. Noviembre-Diciembre 2005. ISSN: 10249435.

[15] C. Soto y C. Barrios. "Gestión del conocimiento. Parte II. Modelo de gestión por procesos". Acimed. Vol. $14 \mathrm{~N}^{\circ}$ 3, pp. 1-16. Mayo-Junio 2006. ISSN: 10249435.

[16] M. McEIroy. "The Second Generation of Knowledge Management". The A/ew Knowledge Management: Complexity, Learning, and Sustainable Innovation: Extract. 2002. Fecha de Consulta: 30 Agosto 2012. URL: http://www.macroinnovation.com/ images/McElroy_On_2ndGenKM.pdf

[17] J.J. Goñi. "Modelo Dinámico de Gestión del conocimiento". La rotación del conocimiento. Fecha de Consulta: 22 de febrero 2012. URL: http://www.gestiondelconocimiento.com/ leer.php?colaborador=jjgoni\&id=166 
[18] L. Kerschberg. "Knowledge management: managing knowledge resources for the intelligent enterprise". Memorias del XXIII Taller de Ingeniería de Sistemas. Santiago, Chile. Julio 2000.

[19] M. Llarena. "Métricas para medir la calidad de la propuesta didáctica de un curso virtual. Fecha de Consulta: 22 de octubre 2012. URL: http://sedici.unlp.edu.ar/bitstream/
handle/10915/18427/Documento_completo. pdf?sequence $=1$

[20] R. Pressman. "Ingeniería del Software, Un enfoque práctico". McGrawHill. 1998.

[21] G. Booch, J. Rumbaugh y I. Jacobson. "El lenguaje Unificado de Modelado 2.0". Segunda edición. Pearson/addi. 2006. ISBN: 9788478290765. 